\title{
Outcomes of Single Bundle Arthroscopic Anterior Cruciate Ligament Reconstruction in a Limited Resource Setting.
}

\section{Binod Sherchan}

National Trauma Center, National Academy of Medical Sciences

Saroj Rai ( $\nabla$ mesaroz@outlook.com )

National Trauma Center, National Academy of Medical Sciences https://orcid.org/0000-0001-68963928

\section{Siddhartha Dhungana}

National Trauma Center, National Academy of Medical Sciences

Nira Tamang

National Trauma Center, National Academy of Medical Sciences

Laxmi Kanta Sharma

National Trauma Center, National Academy of Medical Sciences

Rudra Prasad Marasini

National Trauma Center, National Academy of Medical Sciences

Janith Lal Singh

National Trauma Center, National Academy of Medical Sciences

Kiran Khanal

National Trauma Center, National Academy of Medical Sciences

\section{Research Article}

Keywords: Arthroscopy, Anterior cruciate ligament, Developing country, Urban group, Rural group,

Posted Date: June 26th, 2020

DOI: https://doi.org/10.21203/rs.3.rs-37653/v1

License: (c) (1) This work is licensed under a Creative Commons Attribution 4.0 International License. Read Full License

Version of Record: A version of this preprint was published at Journal of ISAKOS: Joint Disorders \& Orthopaedic Sports Medicine on November 3rd, 2020. See the published version at https://doi.org/10.1136/jisakos-2020-000500. 


\section{Abstract}

Purpose: Despite various challenges, orthopaedic sports surgeons are still providing the arthroscopic service in developing countries like Nepal; however, it is hardly being reported. The main purpose of this study was to compare the clinical outcomes and complications of patients undergoing arthroscopic ACLR in the Urban group and that of the Rural group.

Methods: We evaluated a total of 194 patients, including 98 patients in the Urban group and 96 patients in the Rural group, undergoing arthroscopic ACLR between August 2015 and February 2018. The subjective evaluations were performed using the Tegner-Lysolm score and International Knee Documentation Committee (IKDC) form. The laxity assessments were performed using the Lachman test and the Pivot-shift test. The functional evaluation included the ROM, single-leg hop test, and overall IKDC score. Radiological assessment was performed according to the IKDC guidelines. SPSS was used for data analysis.

Results: There was statistically significant differences in the subjective assessments between 2 groups. No statistically significant differences existed between 2 groups in terms of Laxity and Functional assessments. However, the proportion of laxity, in terms of Lachman test and Pivot-shift test, was higher in the young and active individuals and the proportion of abnormal and severely-abnormal in all parameters of functional outcomes was higher in the older female in the Rural group. Graft failure occurred in 19(17.6\%) knees in the Urban group and 17(16.8\%) knees in the Rural group. Graft failure in the urban group was higher in young and active male patients, whereas failure in the Rural group was more in female patients. Similarly, overall infection occurred in $13(6.2 \%)$ knees, including $5(2.3 \%)$ deep infections and $8(3.8 \%)$ superficial infections.

Conclusion: We advised the similar rehabilitation protocol for all the patients; however, the outcomes were significantly lower in patients living in rural areas as they are found to have poor compliance with the rehabilitation protocol. The overall graft failure rate was $17.2 \%$; however, the reoperation rate was higher in the Urban group than the Rural group. The rate of deep infection was higher in the Rural group as compared to the Urban group.

\section{What Are The New Findings?}

- The commonest etiology was the Road Traffic Accident, and patients from the rural area presented to the hospital for definitive treatment significantly later.

- Patients from rural area showed significantly poor patient-reported outcomes.

- Laxity proportion was more common in young and active males, whereas the stiffness and pain were more common in rural women.

- The overall graft failure and infection rates were $17.2 \%$ and $6.2 \%$, respectively, and the revision rate was higher in the Urban group. 


\section{Introduction}

The most common cause of anterior cruciate ligament (ACL) tear is sports-related injuries ${ }^{1}$. However, it can be injured by a road traffic accident (RTA) and even with normal household activities. About 250,000 people suffer ACL tear in the United States every year, and the number is still in increasing trends ${ }^{2}$. Arthroscopic anterior cruciate ligament reconstruction (ACLR) has been the gold standard and the most effective treatment method with a high success rate ${ }^{3}$.

Among the available autograft, hamstring tendon ( $\mathrm{HT})$ has been one of the most popular graft materials, and satisfactory clinical outcomes can be achieved with a successful ACLR ${ }^{14}$. Several previous studies did not find any significant difference between the HT autograft and BPTB autograft ${ }^{5-7}$. However, the HT has an inherent limitation of being soft tissue graft as it has to undergo ligamentization, which results in apparent laxity ${ }^{2}$. Also, there is a chance of delayed graft incorporation, resulting in early failure ${ }^{4}$. Regardless of such drawbacks, HT has been widely used in clinical practice because of biomechanical superiority, easy to harvest, and minimal donor site morbidity as compared to bone-patellar tendon-bone (BPTB) autograft ${ }^{8}$.

The development of social media, health awareness, and implementation of health insurance policies by the government of Nepal, has led the patient to access the health-care facilities for the definitive treatment for conditions like ACL tear ${ }^{9}$. Moreover, arthroscopy and sports medicine has also gained popularity amongst orthopaedic surgeons recently. However, both the patients and the clinicians are still facing financial, technical, and logistic challenges. Despite these challenges, orthopaedic-sports surgeons are still providing the best possible care to the patients of all the geographical areas of the country. Among the 2.8 million population, about $80 \%$ live in rural Nepal, having agriculture as their primary profession, and only $20 \%$ of people live in the cities ${ }^{10}$. These two groups of patients might have different clinical outcomes and expectations.

We aimed to compare the clinical outcomes and complications of patients undergoing ACLR in the urban area (Urban group) and that of rural area (Rural group). We hypothesized that the clinical outcomes and complications after ACLR in the Rural group are poorer than in the Urban group.

\section{Materials And Methods}

\section{Patients}

We retrospectively evaluated the patients who underwent ACLR using hamstring tendons between August 2015 and February 2018. A total of 275 eligible patients were shortlisted for further evaluation. The information of the patients, including contact details, demographic data, time interval from the injury to surgery, graft diameter, date of surgery, etiology of ACL tear, any associated meniscal and collateral ligament injury status and their surgical procedures, graft tissue used, and any complications, were also 
recorded. After a detailed study of operation notes and radiographic pictures of all the shortlisted patients, 239 patients met the inclusion criteria (Table 1).

Eligible shortlisted patients were contacted via a telephone call and social media to visit the hospital for the final follow-up. Of 239 patients, 98 patients (Male 63, Female 35) in the Urban group and 96 patients (Male 58, Female 38) in the Rural group, with an average age of 30 years, were responded to our calls and visited the hospital for final evaluation

\section{Preoperative preparation and Surgical procedure}

All the patients underwent routine preoperative imaging, including plain radiographs (anteroposterior (AP) and lateral view) and magnetic resonance imaging (MRI), to evaluate the bony and soft tissue status of the knee.

Surgeries were performed under spinal anaesthesia in the supine position using a standard anteromedial portal (AMP) technique. We routinely used, Semitendinosus (ST) and Gracillis (GT), both tendons to make the graft size of $8 \mathrm{~mm}$ or more. The graft was fixed with an Endobutton on the femoral side, whereas a bioabsorbable interference screw on the tibial side. The reparable meniscus was repaired using either the outside-in technique with the suture-shuttle technique or all-inside technique with FASTFIX ${ }^{\mathrm{TM}}$. The partial meniscectomy was performed for irreparable menisci.

\section{Postoperative Rehabilitation}

Postoperative rehabilitation was started on the first postoperative day and dependent upon the meniscal procedure's status. On the first postoperative day, the patient was advised for the ankle pump, quadriceps strengthening, and hamstring stretch exercises. If the patient did not require meniscus repair, then he/she was advised for the gradual ROM with the aim to achieve $120^{\circ}$ in 6 weeks. Weight-bearing, as tolerated, was also advised. However, knee ROM and weight-bearing were avoided for 6 weeks if the patient underwent meniscal repair. Patients were advised for ambulation with crutches or canes. Such assistive devices were continued until the gait became normal. Balance and proprioception training was initiated once the gait became normal (8 weeks). Patients were advised for jogging, running, and household activities at 3 months and return to farm/agriculture work and sports at 12 months.

\section{Clinical Evaluation}

Before the data collection, written informed consent was obtained from the participants. To avoid the susceptibility of bias, a Ph.D. scholar SR performed all the clinical evaluation as he was not involved in the surgery. The clinical evaluation included subjective evaluation and objective evaluation. The subjective evaluations were performed using the Tegner-Lysolm score ${ }^{11}$ and International Knee Documentation Committee (IKDC) form ${ }^{12}$. The laxity assessments were performed using the Lachman 
test and the Pivot-shift test. The functional evaluation included the ROM, single-leg hop test, and overall IKDC score.

\section{Radiological assessment}

In every follow-up visit, operated knees were evaluated for any osteoarthritic changes on weight-bearing $A P$, lateral, and skyline view as per IKDC guidelines ${ }^{12}$. The results were rated as normal, near-normal, abnormal, and severely-abnormal.

\section{Statistical Analysis}

We used IBM SPSS Statistics version 25 for data analysis. Categorical variables were analyzed using the Chi-square test and Fischer's Exact test, and results were presented as frequencies and percentages. Continuous variables were analyzed using independent t-test (two-tailed), and results were presented as mean \pm standard deviation (SD). A difference in a $p$-value of $<0.05$ was considered as statistically significant.

\section{Results}

\section{General results}

Table 2 shows the baseline details of patients. There were no statistically significant differences between the Urban group and Rural group in terms of age, gender, BMI, side of involvement, graft size, follow-up, and associated meniscal injury. However, there were statistically significant differences between the two groups regarding time interval from injury to surgery $(p<0.001)$ and the etiology $(p=0.002)$. Similarly, there was no significant difference in the rate of meniscal injury $(p=0.550)$. There was no statistically significant difference between both the groups in terms of meniscus procedure $(p=0.065)$. None of the patients underwent total meniscectomy.

\section{Clinical results}

Statistically significant differences were observed between the two groups in terms of subjective evaluations at the final follow-up (Table 3). The average IKDC score for the Urban group and Rural group was 85 points and 81 points, respectively $(p=0.002)$. Similarly, the average Tegner-Lysolm score for the Urban group and Rural group was 88 points and 85 points, respectively $(p=0.006)$. The two groups did not show any significant differences statistically regarding the Lachman test $(p=0.423)$ and the Pivotshift test $(p=0.523)$ (Table 4$)$. However, the laxity proportion was higher in the young and active individuals. Both the group did not show any statistically significant differences regarding the ROM, single-leg hop test, and the overall IKDC score (Table 5). However, the proportion of abnormal and severely-abnormal in all the parameters of functional outcomes was more in the older female in the Rural group. 


\section{Radiological results}

According to IKDC radiographic evaluation of weight-bearing AP radiograph, 73 (74.5\%) patients had normal, $21(21.4 \%)$ patients had near-normal, and $4(4.1 \%)$ patients had abnormal radiographic changes in the Urban group, whereas 66 (68.8\%) patients had normal, 21 (21.9\%) had near-normal, 7 (7.3\%) had abnormal, and only $2(2.1 \%)$ patient had severely-abnormal radiographic changes in the Rural group.

\section{Complications}

We included all the patients undergoing isolated ACLR in complications (Table 6); however, the revision ACLR was excluded from the study. There was no significant difference between the two groups. Graft failure occurred in 19(17.6\%) knees in the Urban group and 17(16.8\%) knees in the Rural group. Graft failure in the urban group was higher in young and active male patients, whereas failure in the Rural group was more in female patients. Among the ACL retear, 6(5.6\%) patients in the Urban group and 2(2\%) in the Rural group underwent revision ACLR. The overall infection occurred in $13(6.2 \%)$ knees, including $5(2.3 \%)$ deep infections and 8(3.8\%) superficial infections. All deep infection underwent arthroscopic debridement and lavage with antibiotics within 6 weeks. The graft was removed in 1 knee in each group. Superficial infections healed completely with regular dressing and oral antibiotics. Other complications include anserine bursitis, paresthesia over the anterolateral aspect of the proximal leg, instrument breakage.

\section{Discussion}

The most important findings of our study were that subjective outcomes, including the Tegner-Lysolm and IKDC scores, were significantly lower in the Rural group than in the Urban group. However, the objective findings were not different statistically. The overall graft failure rate was $17.2 \%$; however, the reoperation rate was higher in the Urban group than the Rural group. The rate of deep infection was higher in the Rural group (3\%) vs. Urban group (1.8\%).

In the past years, because of poor socioeconomic status of the patients and lack of expertise, the condition like ACL tears was either unrecognized or neglected in Nepal. The patients used to stay home crippled. The development of social media, health awareness and implementation of health insurance policies by the government of Nepal has led the patient to access the health care facilities for the definitive treatment of such a debilitating condition ${ }^{13}$. Also, arthroscopy and sports medicine, as a subspecialty, have gained popularity amongst young orthopaedic surgeons as minimally invasive surgery. Orthopaedic surgeons are being trained from both the national and international training centers. However, both the patients and the clinicians are still facing technical, financial, and logistic challenges. Despite these challenges, orthopedics and sports surgeons are still providing the best possible care to the patients of all the geographical areas of the country within the limited recourses. 
Unlike in the developed world where the etiology is mostly the sports-related injuries, the primary etiology of the ACL tear in our patients was RTA that consisted of $53 \%$, mostly being two-wheelers accidents. The reason for being RTA as the primary cause of ACL injury is the poor road conditions, poor road engineering, narrow and slippery mountain roads, overcrowded automobiles in the city, low safety barriers and driver's negligence, etc. ${ }^{14,15}$. In the Rural population, farm work or household work was the second most common cause of ACL injury (32\%). In contrast, in the Urban group, the second most common cause was sports-related injury (26\%).

Our patients have not only differences in the etiology, but also they come to the hospital for the definitive treatment only when they have severe pain and significant instability ${ }^{16}$. The main reason for coming late to the hospital is the low economic condition as most of the payments, including the cost of surgery and implants, have to make out of their pocket, and is very expensive ${ }^{16}$. To have their surgery done, even, the patient has to sell their property, and even some family members go abroad, mostly in India and Gulf countries for earning ${ }^{1718}$. Unfortunately, most of the insurance policy does not cover the cost of the implants ${ }^{19}$. As of this study, both the groups presented to the hospital significantly later (average, 20 months in Rural group and 10 months in the Urban group) than what we usually see in the developed world ${ }^{1620}$. Only $39(20 \%)$ of the patients presented to the hospital within 3 months of trauma for definitive surgery.

Even though most of our patients are living in the Rural area, and they are not the sportsperson, we would assume that they are a unique group of patients with a high demand for surgery. Because most of the patients living in the hilly-mountain have to work on the farm, and each of their daily activities is pivoting activities (Figure 1).

Regardless of previous findings of having no differences in the subjective outcome when compared with early and delayed ACLR patients 2021 , our patients had significantly poor subjective outcomes in the Rural group. These differences are probably because of the delayed presentation to the hospital, as many of the patients have already developed some degenerative changes in the cartilage and the meniscus, resulting in pain ${ }^{1622-24}$. That could also be shown by the postoperative radiograph of the patients where $9 \%$ of the patients had abnormal and severely-abnormal radiographic changes in the Rural group vs. $4 \%$ in the Urban group.

AP and rotational laxity measurements were not different significantly between two groups. However, both groups showed a high proportion of laxity in the young and active male patients; it might be associated with early return to activities during graft ligamentization, as one of the global drawbacks of the soft tissue graft 22526 .

For any ligament reconstruction, strict rehabilitation protocol is mandatory for better postoperative outcomes. We counseled all the patients regarding the risk and benefit of the surgery, possible clinical outcomes, and the role of rehabilitation protocol before the surgery. 
We performed ACLR for at least 16 patients, especially females, from the Rural area because of multiple episodes of giving away, resulting in frequent fall from height and even some reported previous fractures of extremities. Although there was no significant difference between the two groups regarding functional outcomes, marked reduction in overall IKDC score, single-leg hope test, and ROM in the Rural group, especially in the female patients, was evident. Twenty-eight percent of patients in the Rural group and 21 $\%$ of patients in the Urban group had abnormal and severely-abnormal single-leg hop test, and $24 \%$ in the Rural and $21 \%$ in the Urban group had abnormal and severely-abnormal overall IKDC score. The proportion of reduced ROM was also more in the Rural group. The discrepancy in functional outcomes is directly related to socioeconomic status and education level of the patients living in Rural area, which directly affects inadequate rehabilitation and early return to work. We prescribed all the patients with similar rehabilitation protocol and upon discharge from the hospital, all the patients were provided with a pamphlet for the instruction of rehabilitation; however, postoperative compliance for the rehabilitation was poor for our patients. The reason for a higher proportion of functional outcomes in the Urban group was a relatively younger age and motivated to the strict rehabilitation protocol.

Graft failure is defined as the presence of recurrent instability, stiffness, and persistent pain ${ }^{27}$. Various etiological factors are associated with graft failure, including patients factors, technical factors, optimum selection of graft, and the inherent limitation of graft tissue used ${ }^{328}$. In a prospective study by Magnussen et al. ${ }^{1}$, the authors reported that the graft size of $8 \mathrm{~mm}$ or less in patients under 20 years of age was found to be the predictor for revision. Similarly, Kamien et al. ${ }^{4}$ found that the age group of less than 25 years had significantly higher hamstring graft failure rate as compared to age above 25 years. They did not find any differences in activity level and graft size. Barret et al. ${ }^{29}$ reported a similar result with hamstring tendon grafts, so they recommended the utilization of bone-patellar tendon-bone graft in young and active individuals.

We defined the Lachman test of Grade 2 or grade 3 with the presence of the Pivot-shift test as a clinical failure. In our series, the overall failure rate was $17.2 \%$, and most of the failure occurred within a year of ACL. The proportion of grade 2 and 3 Lachman test was higher in young and active individuals in both groups. However, the stiffness and pain were more evident in the rural population.

Asian people are relatively shorter stature, so the graft is. However, we insisted on every graft size to make at least $8 \mathrm{~mm}$. As of previous reports, at least $2 \mathrm{~mm}$ of graft in each tunnel must be there in each tunnel for biological incorporation ${ }^{30} 31$. Because of the surgeon's preferences of making an $8 \mathrm{~mm}$ graft, the graft length might obviously be shorter in the tunnels and affected for biological incorporation. Sixty-five percent of all failure was within 6 months in our group, and mostly in active young male individuals. The short graft might have slipped off the interference screw, or some failure might have attributed to the screw divergence, causing early failure. Such prediction led us to use a suture post on the tibial side often if there is any doubt for adequate fixation.

Postoperative infection following ACLR is considered to be the dreadful complication and ranges from $0.14 \%-1.7 \%{ }^{32}$. However, the overall infection rate in our study was $6.2 \%$, with $2.3 \%$ deep infection 
requiring arthroscopic debridement with antibiotic lavage. There might be various reasons for postoperative infection in our cases. Number one is the frequent use of the same set of instruments multiple times on the same day that possibly compromised the sterilization process. Another reason is because of the limited number of operating rooms available in high volume tertiary hospital, the operating room must be used for multiple purposes, including frequent use for debridement of infected cases. Because of the high infection rate, we started presoaking the graft in antibiotic (Vancomycin) solution.

Despite a single experienced orthopaedic surgeon performed all the clinical examinations, who have not involved in the surgery, to avoid the inter-observer bias, various limitations exist in our study. This study has all the biases that a retrospective, non-randomized study would have. We prescribed a similar rehabilitation for all the patients; however, many patients did not attend the complete course because of various reasons, as mentioned above, which might have directly affected the clinical outcomes of the patients. There might be an institutional bias as this study is from a single government hospital where mostly the economically poor patients come for the treatment. Lastly, laxity assessment was performed manually, measurement of laxity using KT-1000 or KT-2000 or other objective methods was not available in our center.

\section{Conclusion}

We advised the similar rehabilitation protocol for all the patients; however, the outcomes were significantly lower in patients living in rural areas as they are found to have poor compliance with the rehabilitation protocol. The overall graft failure rate was $17.2 \%$; however, the reoperation rate was higher in the Urban group than the Rural group. The rate of deep infection was higher in the Rural group as compared to the Urban group.

\section{Abbreviations}

ACL: Anterior cruciate ligament

ACLR: Anterior cruciate ligament reconstruction

AMP: Anteromedial portal

AP: Anteroposterior

BMI: Body mass index

BPTB: Bone-patellar tendon-bone

GT: Gracillis tendon

HT: Hamstring tendon 
IKDC: International Knee Documentation Committee

MRI: Magnetic resonance imaging

ROM: Range of motion

RTA: Road traffic accident

SD: Standard deviation

ST: Semitendinosus tendon

\section{Declarations}

The study was approved by the ethical review committee of the National Academy of Medical Sciences, consent to publish photo was obtained from the participant and all authors declare that no competing interest exist in this research.

\section{References}

1. Magnussen RA, Lawrence JTR, West RL, et al. Graft size and patient age are predictors of early revision after anterior cruciate ligament reconstruction with hamstring autograft. Arthrosc $-\mathrm{J}$ Arthrosc Relat Surg 2012;28(4):526-31.

2. Rai S, Jin SY, Rai B, Tamang N, et al. A Single Bundle Anterior Cruciate Ligament Reconstruction (ACL-R) Using Hamstring Tendon Autograft and Tibialis Anterior Tendon Allograft: A Comparative Study. Curr Med Sci 2018;38(5):818-26.

3. Samitier G, Marcano Al, Alentorn-Geli E, et al. Failure of anterior cruciate ligament reconstruction. Arch Bone Jt Surg 2015;3(4):220-40.

4. Kamien PM, Hydrick JM, Replogle WH, et al. Age, graft size, and tegner activity level as predictors of failure in anterior cruciate ligament reconstruction with hamstring autograft. Am J Sports Med 2013;41(8):1808-12.

5. Maletis GB, Cameron SL, Tengan JJ, et al. A prospective randomized study of anterior cruciate ligament reconstruction: A comparison of patellar tendon and quadruple-strand semitendinosus/gracilis tendons fixed with bioabsorbable interference screws. Am J Sports Med 2007;35(3):384-94.

6. Sajovic $\mathrm{M}$, Vengust $\mathrm{V}$, Komadina $\mathrm{R}$, et al. A prospective, randomized comparison of semitendinosus and gracilis tendon versus patellar tendon autografts for anterior cruciate ligament reconstruction: Five-year follow-up. Am J Sports Med 2006;34(12):1933-40.

7. Samuelsson K, Andersson D, Karlsson J. Treatment of anterior cruciate ligament injuries with special reference to graft type and surgical technique: an assessment of randomized controlled trials. Arthrosc - J Arthrosc Relat Surg 2009;25(10):1139-74. 
8. Sun $\mathrm{K}$, Zhang J, Wang $Y$, et al. Arthroscopic anterior cruciate ligament reconstruction with at least 2.5 years' follow-up comparing hamstring tendon autograft and irradiated allograft. Arthrosc - $\mathrm{J}$ Arthrosc Relat Surg 2011;27(9):1195-202.

9. Magar A. Envisaging beyond community-based health insurance in Nepal. J Nepal Health Res Counc 2013 Sep;11(25):I-II.

10. The World Bank. https://data.worldbank.org/indicator/SP.RUR.TOTL.ZS. 2018.

11. Tegner Y, Lysholm J. Rating systems in the evaluation of knee ligament injuries. Clin Orthop. 1985 Sep;(198):43-9.

12. Irrgang J, Anderson A, Boland,A, et al. Development and Validation of the International Knee Documentation Committee Subjective Knee Form. Am J Sports Med 2001; 29(5): 600-13.

13. Health Insurance Board. https://hib.gov.np/en/. 2020.

14. Huang L, Adhikary KP, Choulagai BP, et al. Road traffic accident and its characteristics in kathmandu valley. J Nepal Med Assoc 2016;55(203):01-6.

15. Shrestha BK. Road traffic accidents in Kathmandu Valley. Third Pole J Geogr Educ 2014;13:54-6.

16. Salzler MJ, Akgün U, Karahan M, et al. Early versus delayed ACL reconstruction: why delayed surgery is our preferred choice. In: Nakamura N, Zaffagnini S, Marx RG, Musahl V, editors. Controversies in the Technical Aspects of ACL Reconstruction: An Evidence-Based Medicine Approach. Berlin, Heidelberg: Springer Berlin Heidelberg; 2017; p. 27-33.

17. Malla B, Rosenbaum MS. Understanding Nepalese labor migration to gulf countries. J Poverty 2017; 3;21(5):411-33.

18. Regmi $P$, van Teijlingen E, Simkhada P, et al. Nepali migrant workers in India. 2017 Available from: http://rgdoi.net/10.13140/RG.2.2.17927.19361

19. Ranabhat CL, Kim C-B, Singh A, et al. Challenges and opportunities towards the road of universal health coverage (UHC) in Nepal: a systematic review. Arch Public Health 2019 Feb 4; 77. https://www.ncbi.nlm.nih.gov/pmc/articles/PMC6360747/

20. Smith TO, Davies L, Hing CB. Early versus delayed surgery for anterior cruciate ligament reconstruction: A systematic review and meta-analysis. Knee Surg Sports Traumatol Arthrosc 2010;18(3):304-11.

21. Meighan AAS, Keating JF, Will E. Outcome after reconstruction of the anterior cruciate ligament in athletic patients. J Bone Jt Surg - Ser B 2003;85(4):521-4.

22. Anderson $\mathrm{AF}$, Anderson $\mathrm{CN}$. Correlation of meniscal and articular cartilage injuries in children and adolescents with timing of anterior cruciate ligament reconstruction. Am J Sports Med 2015;43(2):275-81.

23. Church S, Keating JF. Reconstruction of the anterior cruciate ligament. Timing of surgery and the incidence of meniscal tears and degenerative change. J Bone Jt Surg - Ser B 2005;87(12):1639-42.

24. Dunn WR, Lyman S, Lincoln AE, et al. The effect of anterior cruciate ligament reconstruction on the risk of knee reinjury. Am J Sports Med 2004;32(8):1906-14. 
25. Janssen RPA, Scheffler SU. Intra-articular remodelling of hamstring tendon grafts after anterior cruciate ligament reconstruction. Knee Surg Sports Traumatol Arthrosc Off J ESSKA 2014;22(9):2102-8.

26. Mehran N, Moutzouros V (Bill), Bedi A. A Review of Current Graft Options for Anterior Cruciate Ligament Reconstruction: JBJS Rev 2015 Nov;3(11):1.

27. Johnson DL, Fu FH. Anterior cruciate ligament reconstruction: why do failures occur? Instr Course Lect 1995;44:391-406.

28. Park SY, Oh H, Park S, et al. Factors predicting hamstring tendon autograft diameters and resulting failure rates after anterior cruciate ligament reconstruction. Knee Surg Sports Traumatol Arthrosc 2013;21(5):1111-8.

29. Barrett AM, Craft JA, Replogle WH, et al. Anterior cruciate ligament graft failure: A comparison of graft type based on age and tegner activity level. Am J Sports Med 2011;39(10):2194-8.

30. Leonard JP. Editorial Commentary: My Harvested Hamstring Autograft Is Too Small. Now What? Arthrosc - J Arthrosc Relat Surg 2019;35(5):1555-6.

31. Mariscalco MW, Magnussen RA, Mitchell J, et al. How much hamstring graft needs to be in the femoral tunnel? A MOON cohort study. Eur Orthop Traumatol 2015;6(1):9-13.

32. Stucken C, Garras DN, Shaner JL, et al. Infections in Anterior Cruciate Ligament Reconstruction. Sports Health 2013;5(6):553-7.

\section{Tables}

Table 1. Inclusion and Exclusion criteria

Inclusion criteria

1) Unilateral single bundle primary ACLR using hamstring tendon

2) Associated meniscus tear and meniscal procedure

3) Associated grade I/II MCL tear

4) Previous diagnostic arthroscopy

5) Follow-up period of 2 years or more

6) All age groups

7) All activity levels

\section{Exclusion Criteria}

1) ACLR using other than hamstring graft

2) Multiligamentous injury

3) Previous ACL repair

4) Revision ACLR

5) PCL injury/reconstruction

6) Posterolateral corner (PLC) injury

7) Contralateral ACL injury/reconstruction

ACLR: Anterior Cruciate Ligament Reconstruction; MCL: Medial Collateral Ligament; PCL: Posterior Cruciate Ligament; 
Table 2. Baseline details of the patients (mean \pm SD or $n \%$ ) 


\begin{tabular}{|c|c|c|c|}
\hline Parameters & Urban $(n=98)$ & Rural $(n=96)$ & $p$-value \\
\hline Age (years) & $29.23 \pm 9.88(16-56)$ & $30.95 \pm 7.90(20-54)$ & 0.163 \\
\hline \multicolumn{4}{|l|}{ Gender } \\
\hline Male & $63(64.3)$ & $58(60.4)$ & 0.657 \\
\hline Female & $35(35.7)$ & $38(39.6)$ & \\
\hline BMI & $23.97 \pm 2.93(17-30)$ & $23.74 \pm 3.05(18-30)$ & 0.598 \\
\hline Leg & & & 0.388 \\
\hline Right & $42(42.9)$ & $48(50)$ & \\
\hline Left & $56(57.1)$ & $48(50)$ & \\
\hline Time interval from injury (months) & $9.86 \pm 8.59(1-45)$ & $20.71 \pm 17.01(1-72)$ & $<0.001^{*}$ \\
\hline Graft size & $8.39 \pm 0.47(8-10)$ & $8.32 \pm 0.44(8-10)$ & 0.228 \\
\hline Follow-up (months) & $36.18 \pm 6.56(24-50)$ & $34.30 \pm 7.21(24-48)$ & 0.059 \\
\hline Etiology & & & $0.002^{\star}$ \\
\hline Sports injury & $26(26.5)$ & $21(21.9)$ & \\
\hline Road traffic accident & $61(62.2)$ & $43(44.8)$ & \\
\hline Work (Household/Farm) & $11(11.2)$ & $31(32.3)$ & \\
\hline Other/unknown & $0(0)$ & $1(1)$ & \\
\hline Associated meniscal injury & & & 0.550 \\
\hline Both menisci & $14(14.3)$ & $21(21.9)$ & \\
\hline Medial meniscus & $42(42.9)$ & $38(39.6)$ & \\
\hline Lateral meniscus & $16(16.3)$ & $16(16.7)$ & \\
\hline None & $26(26.5)$ & $21(21.9)$ & \\
\hline Meniscal procedure & & & 0.065 \\
\hline None & $26(26.5)$ & $21(21.9)$ & \\
\hline Meniscal repair & $37(37.8)$ & $25(26)$ & \\
\hline Partial meniscectomy & $35(35.7)$ & $50(52.1)$ & \\
\hline
\end{tabular}


Table 3. Patient reported outcomes of the patients ((mean $\pm S D$ or $n)$

\begin{tabular}{|lccc|}
\hline Parameters & Urban $(n=98)$ & Rural $(n=96)$ & p-value \\
\hline IKDC score & $85.30 \pm 10.54(59-100)$ & $81.87 \pm 10.21(51-100)$ & $0.022^{*}$ \\
\hline Tegner-Lysholm score & $88.26 \pm 8.53(66-100)$ & $84.82 \pm 8.53(57-100)$ & $0.006^{*}$ \\
\hline $\begin{array}{l}\text { IKDC: International Knee Documentation Committee; } n \text { : Number of cases; SD: Standard Deviation; *: } \\
\text { Statistically significant different exists between the groups; }\end{array}$ & \\
\hline
\end{tabular}

Table 4. Laxity assessment of the patients (n, \%)

\begin{tabular}{|lllll|}
\hline Parameters & Grade & Urban $(\mathrm{n}=98)$ & Rural $(\mathrm{n}=96)$ & p-value \\
\hline Lachman test & Grade 0 & $33(33.7)$ & $25(26)$ & 0.423 \\
& Grade 1 & $46(46.9)$ & $54(56.3)$ & \\
& Grade 2 & $16(16.3)$ & $12(12.5)$ & \\
& Grade 3 & $3(3.1)$ & $5(5.2)$ & \\
Pivot-shift test & Grade 0 & $40(40.8)$ & $35(36.5)$ & 0.523 \\
& Grade 1 & $40(40.8)$ & $46(47.9)$ & \\
& Grade 2 & $15(15.3)$ & $10(10.4)$ & \\
& Grade 3 & $3(3.1)$ & $5(5.2)$ & \\
\hline n: Number of cases; & & & \\
\hline
\end{tabular}

Table 5. Functional outcomes of the patients ( $n, \%)$ 


\begin{tabular}{|c|c|c|c|}
\hline Parameters & Urban $(n=98)$ & Rural $(n=96)$ & $\mathrm{p}$-value \\
\hline ROM & & & 0.259 \\
\hline Normal & $80(81.6)$ & $72(75)$ & \\
\hline Near-normal & $15(15.3)$ & $16(16.7)$ & \\
\hline Abnormal & $3(3.1)$ & $8(8.3)$ & \\
\hline Severely-abnormal & 0 & 0 & \\
\hline Single-leg hop test & & & 0.125 \\
\hline Normal & $33(33.7)$ & $20(20.8)$ & \\
\hline Near-normal & $44(44.9)$ & $49(51)$ & \\
\hline Abnormal & $18(18.4)$ & 19 (19.8) & \\
\hline Severely-abnormal & $3(3.1)$ & $8(8.3)$ & \\
\hline Overall IKDC score & & & 0.493 \\
\hline Normal & $26(26.5)$ & $20(20.8)$ & \\
\hline Near-normal & $52(53.1)$ & $53(55.2)$ & \\
\hline Abnormal & $17(17.3)$ & $16(16.7)$ & \\
\hline Severely-abnormal & $3(3.1)$ & $7(7.3)$ & \\
\hline
\end{tabular}

Table 6 Complications ( $\mathrm{n}, \%)$ 


\begin{tabular}{|lcl|}
\hline Parameters & Urban $(\mathrm{n}=108)$ & Rural $(\mathrm{n}=101)$ \\
\hline Graft failure & $19(17.6)$ & $17(16.8)$ \\
Revision & $6(5.6)$ & $2(2)$ \\
No revision & $13(12)$ & $15(14.8)$ \\
\hline Deep Infection & $2(1.8)$ & $3(3)$ \\
Required graft removal & $1(0.9)$ & $1(1)$ \\
No required graft removal & $1(0.9)$ & $2(2)$ \\
\hline Superficial infection & $5(4.6)$ & $3(3)$ \\
\hline Anserine bursitis & $8(7.4)$ & $5(4.9)$ \\
\hline Paresthesia lasting $>6$ months & $11(10.1)$ & $15(14.8)$ \\
\hline Instrument breakage & $1(0.9)$ & $1(1)$ \\
\hline Total & $47(43.5)$ & $44(43.6)$ \\
\hline n: Number of cases & & \\
\hline
\end{tabular}

Figures 


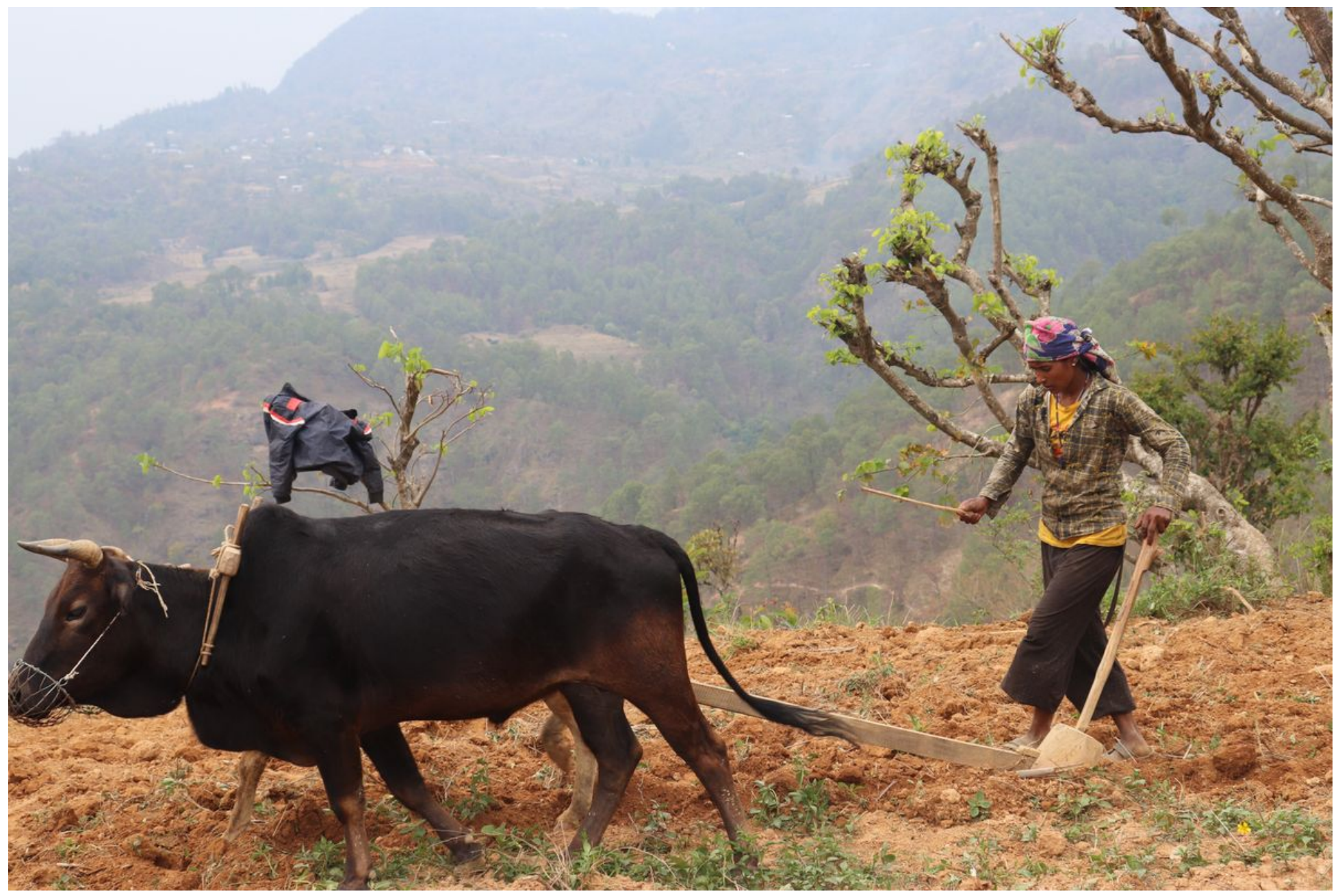

\section{Figure 1}

A woman plowing in the field Rural village of Humla, western Nepal 